\title{
$(0,2)$ Gauged Linear Sigma Model on Supermanifold
}

\author{
Yusuke Okame and Mitsuo J. Hayashi
}

\author{
Tokai University, 1117 Kitakaname, Hiratsuka, Kanagawa 259-1292, Japan \\ E-mail: mhayashi@keyaki.cc.u-tokai.ac.jp
}

\begin{abstract}
We construct $(0,2), D=2$ gauged linear sigma model on supermanifold with both an Abelian and non-Abelian gauge symmetry. For the purpose of checking the exact supersymmetric (SUSY) invariance of the Lagrangian density, it is convenient to introduce a new operator $\hat{U}$ for the Abelian gauge group. The $\hat{U}$ operator provides consistency conditions for satisfying the SUSY invariance. On the other hand, it is not essential to introduce a similar operator in order to check the exact SUSY invariance of the Lagrangian density of non-Abelian model, contrary to the Abelian one. However, we still need a new operator in order to define the $(0,2)$ chirality conditions for the $(0,2)$ chiral superfields. The operator $\hat{U}^{a}$ can be defined from the conditions assuring the $(0,2)$ supersymmetric invariance of the Lagrangian density in superfield formalism for the $(0,2) \mathrm{U}(\mathrm{N})$ gauged linear sigma model.

We found consistency conditions for the Abelian gauge group which assure $(0,2)$ supersymmetric invariance of Lagrangian density and agree with $(0,2)$ chirality conditions for the superpotential. The supermanifold $\mathcal{M}^{m \mid n}$ becomes the super weighted complex projective space $W C P^{m-1 \mid n}$ in the $\mathrm{U}(1)$ case, which is considered as an example of a Calabi-Yau supermanifold. The superpotential $W(\phi, \xi)$ for the non-Abelian gauge group satisfies more complex condition for the $\mathrm{SU}(\mathrm{N})$ part, except the $\mathrm{U}(1)$ part of $\mathrm{U}(\mathrm{N})$, but does not satisfy a quasi-homogeneous condition. This fact implies the need for taking care of constructing the Calabi-Yau supermanifold in the $\mathrm{SU}(\mathrm{N})$ part. Because more stringent restrictions are imposed on the form of the superpotential than in the $U(1)$ case, the superpotential seems to define a certain kind of new supermanifolds which we cannot identify exactly with one of the mathematically well defined objects.
\end{abstract}




\section{Introduction}

Recently, it was reported that the perturbative expansion of the $D=4, \mathcal{N}=4$ super Yang-Mills theory with the $\mathrm{U}(\mathrm{N})$ gauge group is equivalent to the instanton expansion of the topological $B$ model for which the target space is the Calabi-Yau supermanifold $C P^{3 \mid 4}$. The connection between the topological string theory on supermanifold $C P^{3 \mid 4}$ and the $D=4, \mathcal{N}=4$ super Yang-Mills theory is established through the explicit calculations of the Maximally-Helicityviolating (MHV) amplitude that lead the twistor equations [1]. Furthermore the methods for calculating many types of MHV amplitudes which include loop amplitudes were developed [2] [18. From these aspects, the Calabi-Yau supermanifold played an important role to establish the relation between the super Yang-Mills theory and topological B model. Recent works on these relationships have investigated of the nature of the Calabi-Yau supermanifold. The geometry of the Calabi-Yau supermanifold was shown to be related to the curvature of the Grassmann even submanifold[19, 20].

The super Landau-Ginzburg mirror symmetry was used to construct the correspondence between the topological B model on $C P^{3 \mid 4}$ as $\mathrm{D}$ instanton[1], and the topological A model on $C P^{3 \mid 3} \times C P^{3 \mid 3}$ 21, 22]. (These supermanifold are both Calabi-Yau supermanifolds.) These mirror correspondences were proved by defining the superpotential on each case 23. In fact, the restricted superpotential on A (B) model corresponds to the restricted superpotential on B (A) model through the mirror symmetry. These restrictions are given by physical symmetries like supersymmetry. As a simple example, these superpotentials have been shown in the $(2,2) \mathrm{U}(1)$ gauged linear sigma model, because the non-linear sigma model description with Calabi-Yau supermanifold is given by the gauged linear sigma model in its infrared limit [23]. Then the restriction of superpotential became equivalent to the $(2,2)$ supersymmetric invariance of the total $(2,2)$ Lagrangian density.

In [24], the Lagrangian density of $(2,2) \mathrm{U}(1)$ gauged linear sigma model on supermanifold has been constructed. The supermanifold then became the Calabi-Yau supermanifold which was defined by the Calabi-Yau condition [1, 25,

$$
\sum_{I} Q_{I}-\sum_{A} q_{A}=0
$$

However, in Ref. 24 the $(2,2)$ supersymmetric transformation properties of the total $(2,2)$ Lagrangian density seems incomplete, because the superpotential term in 24 is not exactly closed under the $(2,2)$ supersymmetric tansformation when the vector multiplets are included. If we consider the $(2,2) \mathrm{U}(1)$ gauged linear sigma model, the $(2,2)$ supersymmetric transformation must include the $U(1)$ vector multiplets in its transformation. Additionally, in Ref. 24], a Lagrangian density of the $(0,2) \mathrm{U}(1)$ gauged linear sigma model was proposed whose transformation properties under the $(0,2)$ supersymmetric transformation was identified by the $(0,2)$ part of the $(2,2)$ transformation on supermanifold. However, the $\mathrm{U}(1)$ charges of each local coordinates must retain the same values. This result means that the number of Grassmann even coordinates is equal to the number of Grassmann odd coordinates from Eq.(10), as far as we focus on the Calabi-Yau supermanifolds which are defined by the mirror symmetric correspondence with the super Landau-Ginzburg model. In this case, the Calabi-Yau supermanifolds will be ristricted to $C P^{m-1 \mid m}$.

In the present paper, as the first move toward finding out the correspondence between the Calabi-Yau supermanifold and the super Landau-Ginzburg model, we will concentrate on the construction of a consistent theory of the two-dimensional $(0,2) \mathrm{U}(1)$ gauged linear sigma model on a supermanifold. The Lagrangian density of this model becomes $(0,2)$ supersymmetric invariant under the corrected $(0,2)$ supersymmetry which includes the vector multiplets. Then we obtain the restrictions on the superpotential which assure the $(0,2)$ supersymmetric invarinace of Lagrangian density. Furthermore, the conditions define the more general form of the CalabiYau supermanifold, such as $W C P^{m-1 \mid n}$, by using the newly introduced operator $\hat{U}$. Next, we 
will construct a consistent $(0,2) \mathrm{U}(\mathrm{N})$ gauged linear sigma model on supermanifold. We will show that the restrictions on the superpotential are similar to the $\mathrm{U}(1)$ gauged linear sigma model for the $\mathrm{U}(1)$ part of $\mathrm{U}(\mathrm{N})$, while for the $\mathrm{SU}(\mathrm{N})$ part the restrictions seem to be stronger than in the $\mathrm{U}(1)$ gauged liear sigma model on the supermanifolds.

In Section 2, we define supermultiplets of the $D=2,(0,2) \mathrm{U}(1)$ gauged linear sigma model and construct the Lagrangian densities on a supermanifold, where we introduce a new operator in order to distinguish the $\mathrm{U}(1)$ charges of local coordinates on the supermanifold. In Section 3 , we derive the $(0,2)$ supersymmetric invariance of the Lagrangian densities defined in Section 2 , and obtain the restriction imposed on the superpotential. We explicitly define the new operator assumed in Section 2 and describe the implication of this new operator on the $(0,2)$ supersymmetric invariance of the theory. In Section 4, we extend the gauge group to the nonAbelian case and construct the $D=2,(0,2) \mathrm{U}(\mathrm{N})$ gauged linear sigma model Lagrangian densities on a supermanifold. In Section 5 , the $(0,2)$ supersymmetric invariance is verified on the model constructed in Section 4. Then we obtain the restriction on the superpotential in the $\mathrm{U}(\mathrm{N})$ gauge group. In Section 6, the operator introduced in Section 3 is extended to the non-Abelian $\mathrm{U}(\mathrm{N})$ gauge transformation and is shown in relation to the $(0,2)$ supersymmetry invariance.

In Section 7, we summarize and discuss our constructions of the $D=2,(0,2)$ gauged linear sigma models. Our notations are the same as those of [26].

\section{$2 \quad(2,2)$ and $(0,2)$ Supermultiplets}

By introducing several $(0,2)$ superfields, we construct the total $(0,2)$ Lagrangian density by a method similar as used in Ref. 24]. However, in the original method it is impossible to assign different values of the U(1) charge to each local coordinate. In this section we solve this problem by introducing a new operator $\hat{U}$, by which it is possible to assign different values of the $\mathrm{U}(1)$ charge to each local coordinate, and construct the more general form of $(0,2)$ Lagrangian density. Furthermore, by using the new operator $\hat{U}$, we will obtain the more general Calabi-Yau supermanifold, where the number of Grassmann even local coordinates and Grassmann odd local coordinates is different. This distinction was not made in the method of Ref. 24.

The $D=2, \mathcal{N}=2$ superfields are defined on the $(2,2)$ superspace. We herein redefine these superfields on the $(0,2)$ superspace and construct the $(0,2)$ Lagrangian density by using a new operator $\hat{U}$.

In $D=2$, the $(2,2)$ Grassmann even chiral superfield $\Phi_{(2,2)}$ and the $(2,2)$ Grassmann odd chiral superfield $\Xi_{(2,2)}$ are defined as:

$$
\begin{aligned}
& \Phi_{(2,2)}^{I} \\
= & \phi^{I}+\sqrt{2}\left(\theta^{+} \psi_{+}^{I}+\theta^{-} \psi_{-}^{I}\right)+2 \theta^{+} \theta^{-} F^{I}-i \theta^{-} \bar{\theta}^{-} \partial_{-} \phi^{I}-i \theta^{+} \bar{\theta}^{+} \partial_{+} \phi^{I} \\
& -\sqrt{2} i \theta^{+} \theta^{-} \bar{\theta}^{-} \partial_{-} \psi_{+}^{I}+\sqrt{2} i \theta^{+} \theta^{-} \bar{\theta}^{+} \partial_{+} \psi_{-}^{I}-\theta^{+} \theta^{-} \bar{\theta}^{-} \bar{\theta}^{+} \partial_{-} \partial_{+} \phi^{I}, \\
& \Xi_{(2,2)}^{A} \\
= & \xi^{A}+\sqrt{2}\left(\theta^{+} b_{+}^{A}+\theta^{-} b_{-}^{A}\right)+2 \theta^{+} \theta^{-} \chi^{A}-i \theta^{-} \bar{\theta}^{-} \partial_{-} \xi^{A}-i \theta^{+} \bar{\theta}^{+} \partial_{+} \xi^{A} \\
& -\sqrt{2} i \theta^{+} \theta^{-} \bar{\theta}^{-} \partial_{-} b_{+}^{A}+\sqrt{2} i \theta^{+} \theta^{-} \bar{\theta}^{+} \partial_{+} b_{-}^{A}-\theta^{+} \theta^{-} \bar{\theta}^{-} \bar{\theta}^{+} \partial_{-} \partial_{+} \xi^{A},
\end{aligned}
$$

where $\mu=0,3, g_{\mu \nu}=\operatorname{diag}(-1,+1)$, and $\partial_{ \pm}=\partial_{0} \pm \partial_{3}$ [24]. The supermanifold is defined on $\mathcal{M}^{m \mid n},(I=1, \cdots, m, A=1, \cdots, n)$. For the $(2,2)$ chiral superfield, we introduce the operator $\hat{U}$, which satisfies the following relations:

$$
\begin{aligned}
& \hat{U} \Phi_{(2,2)}^{I}=Q_{I} \Phi_{(2,2)}^{I}, \quad \hat{U} \bar{\Phi}_{(2,2)}^{I}=-Q_{I} \bar{\Phi}_{(2,2)}^{I}, \\
& \hat{U} \Xi_{(2,2)}^{A}=q_{A} \Xi_{(2,2)}^{A}, \quad \hat{U} \bar{\Xi}_{(2,2)}^{A}=-q_{A} \bar{\Xi}_{(2,2)}^{A},
\end{aligned}
$$


where $Q_{I}$ and $q_{A}$ are the $\mathrm{U}(1)$ charges of $\Phi_{(2,2)}^{I}$ and $\Xi_{(2,2)}^{A}$, respectively, and the $\hat{U}$ operator is considered to define the $\mathrm{U}(1)$ charges of the superfields. We assume that $\hat{U}$ is a Grassmann even operator that satisfies:

$$
\begin{aligned}
& {\left[\hat{U}, \theta^{\alpha}\right]=\left[\hat{U}, \bar{\theta}^{\alpha}\right]=0,} \\
& \left.\hat{U}, \frac{\partial}{\partial \theta^{\alpha}}\right]=\left[\hat{U}, \frac{\partial}{\partial \bar{\theta}^{\alpha}}\right]=0,
\end{aligned}
$$

where $\alpha= \pm$. We define the covariant derivative of the $(0,2)$ supersymmetric transformation by incorporating the $\hat{U}$ and gauge fields $v_{\mu}$,

$$
\mathcal{D}_{+} \equiv e^{-\Psi \hat{U}}\left(\frac{\partial}{\partial \theta^{+}}-i \bar{\theta}^{+} \partial_{+}\right) e^{\Psi \hat{U}},
$$

where $v_{ \pm}=v_{0} \pm v_{3}$ and $\Psi=\theta^{+} \bar{\theta}^{+} v_{+}$. The $(0,2)$ super charges are defined by incorporating the $\hat{U}$ operator and the gauge fields $v_{\mu}$ as:

$$
\mathcal{Q}_{+} \equiv e^{\Psi \hat{U}}\left(\frac{\partial}{\partial \theta^{+}}+i \bar{\theta}^{+} \partial_{+}\right) e^{-\Psi \hat{U}}
$$

We now consider the $(0,2)$ case. The $(0,2)$ chirality conditions are defined by using Eq. (6) for arbitrary functions $F\left(x_{\mu}, \theta^{+}, \bar{\theta}^{+}\right)$and $\bar{F}\left(x_{\mu}, \theta^{+}, \bar{\theta}^{+}\right)$on the $(0,2)$ superspace:

$$
\mathcal{D}_{+} \bar{F}=\overline{\mathcal{D}}_{+} F=0 \text {. }
$$

We can define the $(0,2)$ chiral superfields that satisfy Eq. (8) from the $(2,2)$ chiral multiplets by imposing restrictions $\theta^{-}=\bar{\theta}^{-}=0[24$.

$$
\begin{aligned}
& \left.\Phi_{(0,2)}^{I} \equiv \Phi_{(2,2)}^{I} e^{Q_{I} \Psi}\right|_{\theta^{-}=\bar{\theta}^{-}=0}, \\
& \left.\Xi_{(0,2)}^{A} \equiv \Xi_{(2,2)}^{A} e^{q_{A} \Psi}\right|_{\theta^{-}=\bar{\theta}^{-}=0},
\end{aligned}
$$

where the covariant derivatives for the $\mathrm{U}(1)$ gauge transformation are given by

$$
D_{\mu}=\partial_{\mu}+i v_{\mu} \hat{U} \text {. }
$$

Since Eqs. (9) and (10) satisfy the $(0,2)$ chirality conditions, they are the $(0,2)$ chiral superfields:

$$
\begin{aligned}
& \overline{\mathcal{D}}_{+} \Phi_{(0,2)}^{I}=\mathcal{D}_{+} \bar{\Phi}_{(0,2)}^{I}=0, \\
& \overline{\mathcal{D}}_{+} \Xi_{(0,2)}^{A}=\mathcal{D}_{+} \bar{\Xi}_{(0,2)}^{A}=0 .
\end{aligned}
$$

Next, we define the Lagrangian density $\mathcal{L}_{k i n}$. for the $(0,2)$ chiral superfields. Denoting the $\theta^{-} \bar{\theta}^{-}$term of the $(2,2)$ vector superfields as $\mathcal{V}$, we have

$$
\mathcal{V}=i v_{-}+2 \theta^{+} \bar{\lambda}_{-}+2 \bar{\theta}^{+} \lambda_{-}+2 i \theta^{+} \bar{\theta}^{+} D
$$

The $\mathrm{U}(1)$ charge for $\mathcal{V}$ is assumed to be zero, i.e.,

$$
\hat{U} \mathcal{V}=0 \text {. }
$$

From the assumptions on U(1) charges for $(0,2)$ chiral superfields in Eqs. (4) and (15), we obtain

$$
\begin{aligned}
& \hat{U} \Phi_{(0,2)}^{I}=Q_{I} \Phi_{(0,2)}^{I}, \quad \hat{U} \bar{\Phi}_{(0,2)}^{I}=-Q_{I} \bar{\Phi}_{(0,2)}^{I}, \\
& \hat{U} \Xi_{(0,2)}^{A}=q_{A} \Xi_{(0,2)}^{A}, \quad \hat{U} \bar{\Xi}_{(0,2)}^{A}=-q_{A} \bar{\Xi}_{(0,2)}^{A} .
\end{aligned}
$$


Using $\mathcal{V}$, we define the covariant derivative for gauge transformation:

$$
\mathcal{D}_{0}-\mathcal{D}_{3} \equiv \partial_{-}+\mathcal{V} \hat{U}
$$

From the $(0,2)$ chiral superfields and Eq. (17), $\mathcal{L}_{k i n}$. is given by

$$
\begin{aligned}
\mathcal{L}_{\text {kin. }}=\frac{i}{2} \int d \theta^{+} d \bar{\theta}^{+} & {\left[\sum_{I} \bar{\Phi}_{(0,2)}^{I}\left(\mathcal{D}_{0}-\mathcal{D}_{3}\right) \Phi_{(0,2)}^{I}\right.} \\
& \left.+\sum_{A} \bar{\Xi}_{(0,2)}^{A}\left(\mathcal{D}_{0}-\mathcal{D}_{3}\right) \Xi_{(0,2)}^{A}\right] .
\end{aligned}
$$

Next, we will define the Lagrangian density $\mathcal{L}_{\text {gauge }}$ and the Fayet-Iliopoulos $(\mathrm{FI})$ term $\mathcal{L}_{D, \theta}$ for the vector superfield $\mathcal{V}$. The gauge invariant field strength $\Upsilon$ is defined as

$$
\Upsilon \equiv \overline{\mathcal{D}}_{+} \mathcal{V}+\theta^{+} \partial_{-} v_{+}
$$

From Eq. (19), the kinetic Lagrangian density $\mathcal{L}_{\text {gauge }}$ of this gauge multiplet is given as

$$
\mathcal{L}_{\text {gauge }}=\frac{1}{8 e^{2}} \int d \theta^{+} d \bar{\theta}^{+} \bar{\Upsilon} \Upsilon
$$

and the FI term is

$$
\mathcal{L}_{D, \theta}=\left.\frac{t}{4} \int d \theta^{+} \Upsilon\right|_{\bar{\theta}^{+}=0}+\left.\frac{\bar{t}}{4} \int d \bar{\theta}^{+} \bar{\Upsilon}\right|_{\theta^{+}=0},
$$

with the FI parameter $t=i r+\theta /(2 \pi)$.

Since the field components in Eq. (19) contain part of the $(2,2)$ vector supermultiplet, the residual field components should be introduced into the theory by

$$
\Omega \equiv \tau+\sqrt{2} i \theta^{+} \bar{\omega}_{+}-i \theta^{+} \bar{\theta}^{+} \partial_{+} \tau
$$

where Eq. (22) is a Grassmann even superfield and assumed to be chargeless, i.e.,

$$
\hat{U} \Omega=\hat{U} \bar{\Omega}=0 .
$$

From Eq. (22), we can define the Lagrangian density $\mathcal{L}_{\Omega}$ as

$$
\mathcal{L}_{\Omega}=\frac{i}{2 e^{2}} \int d \theta^{+} d \bar{\theta}^{+} \bar{\Omega} \partial_{-} \Omega
$$

In order to construct the $(0,2)$ superpotential consitently, we introduce some $(0,2)$ chiral superfield valued functions $E_{a}\left(\Phi_{(0,2)}, \Omega\right), \tilde{E}_{\tilde{a}}\left(\Xi_{(0,2)}, \Omega\right)$ where the indices $a$ and $\tilde{a}$ denote Grassmann even and Grassmann odd, respectively. In addition, the other $(0,2)$ superfields are introduced as

$$
\begin{aligned}
& \Lambda_{-a}^{\prime} \equiv \lambda_{-a}-\sqrt{2} \theta^{+} G_{a}-i \theta^{+} \bar{\theta}^{+} \partial_{+} \lambda_{-a}, \\
& \tilde{\Lambda}_{-\tilde{a}}^{\prime} \equiv \tilde{\lambda}_{-\tilde{a}}-\sqrt{2} \theta^{+} \tilde{G}_{\tilde{a}}-i \theta^{+} \bar{\theta}^{+} \partial_{+} \tilde{\lambda}_{-\tilde{a}} .
\end{aligned}
$$

The U(1) charges for the fields in Eqs. (25) and (26) are assumed as

$$
\begin{array}{ll}
\hat{U} \Lambda_{-a}^{\prime}=\alpha_{a} \Lambda_{-a}^{\prime}, & \hat{U} \bar{\Lambda}_{-a}^{\prime}=-\alpha_{a} \bar{\Lambda}_{-a}^{\prime}, \\
\hat{U} \tilde{\Lambda}_{-\tilde{a}}^{\prime}=\beta_{\tilde{a}} \tilde{\Lambda}_{-\tilde{a}}^{\prime}, & \hat{U} \overline{\tilde{\Lambda}}_{-\tilde{a}}^{\prime}=-\beta_{\tilde{a}} \overline{\tilde{\Lambda}}_{-\tilde{a}}^{\prime} .
\end{array}
$$


Moreover, by Eqs. (25) and (26), we define new fields as

$$
\begin{aligned}
& \Lambda_{-a} \equiv \Lambda_{-a}^{\prime}-\sqrt{2} \bar{\theta}^{+} E_{a}(\Phi, \Omega), \\
& \tilde{\Lambda}_{-\tilde{a}} \equiv \tilde{\Lambda}_{-\tilde{a}}^{\prime}-\sqrt{2} \bar{\theta}^{+} \tilde{E}_{\tilde{a}}(\Xi, \Omega) .
\end{aligned}
$$

We can then define the $(0,2)$ superfields as:

$$
\begin{aligned}
& \Lambda_{-a(0,2)} \equiv \Lambda_{-a} e^{\alpha_{a} \Psi} \\
& \tilde{\Lambda}_{-\tilde{a}(0,2)} \equiv \tilde{\Lambda}_{-\tilde{a}} e^{\beta_{\tilde{a}} \Psi}
\end{aligned}
$$

by using Eqs. (27), (28) and (29). We then obtain the Lagrangian density $\mathcal{L}_{\Lambda}$ from Eqs. (30) and (31).

$$
\mathcal{L}_{\Lambda}=\frac{1}{2} \int d \theta^{+} d \bar{\theta}^{+}\left[\sum_{a} \bar{\Lambda}_{-a(0,2)} \Lambda_{-a(0,2)}+\sum_{\tilde{a}} \overline{\tilde{\Lambda}}_{-\tilde{a}(0,2)} \tilde{\Lambda}_{-\tilde{a}(0,2)}\right]
$$

We need more $(0,2)$ chiral superfield valued functions $J^{a}\left(\Phi_{(0,2)}, \Xi_{(0,2)}\right), \tilde{J}^{\tilde{a}}\left(\Phi_{(0,2)}, \Xi_{(0,2)}\right)$. The $\mathrm{U}(1)$ charges for these fields are assumed as

$$
\begin{aligned}
& \hat{U} J^{a}(\phi, \xi)=\sum_{I} \hat{U} \phi^{I} \frac{\partial J^{a}(\phi, \xi)}{\partial \phi^{I}}+\sum_{A} \hat{U} \xi^{A} \frac{\partial J^{a}(\phi, \xi)}{\partial \xi^{A}} \\
& \hat{U} \tilde{J}^{\tilde{a}}(\phi, \xi)=\sum_{I} \hat{U} \phi^{I} \frac{\partial \tilde{J}^{\tilde{a}}(\phi, \xi)}{\partial \phi^{I}}+\sum_{A} \hat{U} \xi^{A} \frac{\partial \tilde{J}^{\tilde{a}}(\phi, \xi)}{\partial \xi^{A}} .
\end{aligned}
$$

Here we impose the following restrictions on the fields $E_{a}(\phi, \tau), \tilde{E}_{\tilde{a}}(\phi, \tau)$ and $J^{a}(\phi, \xi), \tilde{J}^{\tilde{a}}(\phi, \xi)$ :

$$
\sum_{a} E_{a}(\phi, \tau) J^{a}(\phi, \xi)+\sum_{\tilde{a}} \tilde{E}_{\tilde{a}}(\xi, \tau) \tilde{J}^{\tilde{a}}(\phi, \xi)=0
$$

From these restrictions, we can obtain the $(0,2)$ chirality conditions

$$
\begin{aligned}
& \overline{\mathcal{D}}_{+}\left(\sum_{a} \Lambda_{-a(0,2)} J^{a}\left(\Phi_{(0,2)}, \Xi_{(0,2)}\right)+\sum_{\tilde{a}} \tilde{\Lambda}_{-\tilde{a}(0,2)} \tilde{J}^{\tilde{a}}\left(\Phi_{(0,2)}, \Xi_{(0,2)}\right)\right) \\
= & \mathcal{D}_{+}\left(\sum_{a} \bar{J}^{a}\left(\bar{\Phi}_{(0,2)}, \bar{\Xi}_{(0,2)}\right) \bar{\Lambda}_{-a(0,2)}+\sum_{\tilde{a}} \overline{\tilde{J}}^{\tilde{a}}\left(\bar{\Phi}_{(0,2)}, \bar{\Xi}_{(0,2)}\right) \overline{\tilde{\Lambda}}_{-\tilde{a}(0,2)}\right) \\
= & 0
\end{aligned}
$$

which define $(0,2)$ chiral superfields and provide the Lagrangian density $\mathcal{L}_{J}$ as follows:

$$
\begin{aligned}
& \mathcal{L}_{J}=\frac{1}{\sqrt{2}} \int d \theta^{+}\left[\sum_{a} \Lambda_{-a(0,2)} J^{a}\left(\Phi_{(0,2)}, \Xi_{(0,2)}\right)\right. \\
&+\left.\sum_{\tilde{a}} \tilde{\Lambda}_{-\tilde{a}(0,2)} \tilde{J}^{\tilde{a}}\left(\Phi_{(0,2)}, \Xi_{(0,2)}\right)\right]\left.\right|_{\bar{\theta}^{+}=0} \\
&+\frac{1}{\sqrt{2}} \int d \bar{\theta}^{+} {\left[\sum_{a} \bar{J}^{a}\left(\bar{\Phi}_{(0,2)}, \bar{\Xi}_{(0,2)}\right) \bar{\Lambda}_{-a(0,2)}\right.} \\
&\left.+\sum_{\tilde{a}} \overline{\tilde{J}}^{\tilde{a}}\left(\bar{\Phi}_{(0,2)}, \bar{\Xi}_{(0,2)}\right) \overline{\tilde{\Lambda}}_{-\tilde{a}(0,2)}\right]\left.\right|_{\theta^{+}=0}
\end{aligned}
$$


We now describe the correspondences between the $(2,2)$ field components and the $(0,2)$ field components. First, the following differential operator is defined:

$$
\mathcal{D}_{-} \equiv e^{-\Pi \hat{U}}\left(\frac{\partial}{\partial \theta^{-}}-i \bar{\theta}^{-} \partial_{-}\right) e^{\Pi \hat{U}},
$$

where $\Pi=\theta^{-} \bar{\theta}^{-} v_{-}$. We can then find the following relations:

$$
\begin{aligned}
& \Lambda_{-a}^{\prime} e^{\alpha_{a} \Psi}=\left.\frac{1}{\sqrt{2}} \mathcal{D}_{-}\left(\Phi_{(2,2)}^{I} e^{Q_{I} \Psi}\right)\right|_{\theta^{-}=\bar{\theta}^{-}=0}, \\
& \tilde{\Lambda}_{-\tilde{a}}^{\prime} e^{\beta_{\tilde{a}} \Psi}=\left.\frac{1}{\sqrt{2}} \mathcal{D}_{-}\left(\Xi_{(2,2)}^{A} e^{q_{A} \Psi}\right)\right|_{\theta^{-}=\bar{\theta}^{-}=0} .
\end{aligned}
$$

From these relations, it is shown that $I=a, A=\tilde{a}$ for indices and $Q_{I}=\alpha_{a}, q_{A}=\beta_{\tilde{a}}$ for $\mathrm{U}(1)$ charges. The exact correspondences between the field components of the $(2,2)$ chiral superfield and those of the $(0,2)$ superfields are given as follows:

$$
\begin{aligned}
& \lambda_{-a}=\psi_{-}^{I}, \quad \tilde{\lambda}_{-\tilde{a}}=b_{-}^{A}, \\
& G_{a}=F^{I}, \quad \tilde{G}_{\tilde{a}}=\chi^{A} .
\end{aligned}
$$

In order to find the corresponding relations between the $(2,2)$ superfields and the $(0,2)$ superfields, the products of the $(2,2)$ chiral superfields and the $(2,2)$ twist chiral superfields are shown. The $(2,2)$ twist chiral superfield is defined as:

$$
\begin{aligned}
\Sigma= & \sigma+\sqrt{2} i \theta^{+} \bar{\lambda}_{+}-\sqrt{2} i \bar{\theta} \lambda_{-}+\sqrt{2} \theta^{+} \bar{\theta}^{-}\left(D-i v_{03}\right)+i \theta^{-} \bar{\theta}^{-} \partial_{-} \sigma \\
& -i \theta^{+} \bar{\theta}^{+} \partial_{+} \sigma-\sqrt{2} \theta^{+} \theta^{-} \bar{\theta}^{-} \partial_{-} \bar{\lambda}_{+}+\sqrt{2} \theta^{+} \bar{\theta}-\bar{\theta}^{+} \partial_{+} \lambda_{-} \\
& +\theta^{+} \theta^{-} \bar{\theta}^{-} \bar{\theta}^{+} \partial_{-} \partial_{+} \sigma .
\end{aligned}
$$

We find the correspondences as follows:

$$
\begin{aligned}
& \overline{\mathcal{D}}_{+} \Lambda_{-a(0,2)}=\left.2 Q_{I} \Sigma \Phi_{(2,2)}^{I} e^{Q_{I} \Psi}\right|_{\theta^{-}=\bar{\theta}^{-}=0}, \\
& \overline{\mathcal{D}}_{+} \tilde{\Lambda}_{-\tilde{a}(0,2)}=\left.2 q_{A} \Sigma \Xi_{(2,2)}^{A} e^{q_{A} \Psi}\right|_{\theta^{-}=\bar{\theta}^{-}=0},
\end{aligned}
$$

where we assumed the following relations:

$$
\begin{aligned}
& \hat{U} E_{a}(\phi, \tau)=\sum_{I} \hat{U} \phi^{I} \frac{\partial E_{a}(\phi, \tau)}{\partial \phi^{I}}, \\
& \hat{U} \tilde{E}_{\tilde{a}}(\xi, \tau)=\sum_{A} \hat{U} \xi^{A} \frac{\partial \tilde{E}_{\tilde{a}}(\xi, \tau)}{\partial \xi^{A}} .
\end{aligned}
$$

The correspondences between the field components of the $(2,2)$ chiral superfields and the $(0,2)$ superfields are derived as

$$
\begin{aligned}
& E_{a}(\phi, \tau)=\sqrt{2} Q_{I} \sigma \phi^{I}, \\
& \tilde{E}_{\tilde{a}}(\xi, \tau)=\sqrt{2} q_{A} \sigma \xi^{A}, \\
& \tau=\sigma, \quad \omega_{+}=\lambda_{+} .
\end{aligned}
$$

Finally, we present the following relations between the field components of the $(0,2)$ superfields $J^{a}\left(\Phi_{(0,2)}, \Xi_{(0,2)}\right), \tilde{J}^{\tilde{a}}\left(\Phi_{(0,2)}, \Xi_{(0,2)}\right)$ and those of the $(2,2)$ superfields by using the superpotential $W$ as

$$
\begin{aligned}
& J^{a}(\phi, \xi)=\frac{\partial W(\phi, \xi)}{\partial \phi^{I}}, \\
& \tilde{J}^{\tilde{a}}(\phi, \xi)=\frac{\partial W(\phi, \xi)}{\partial \xi^{A}} .
\end{aligned}
$$


We have shown that the total $(0,2)$ Lagrangian density $\mathcal{L}_{(0,2)}$ is obtained from Eqs. (18), (20), (21), (24), (32), and (37) as follows:

$$
\mathcal{L}_{(0,2)}=\mathcal{L}_{\text {kin. }}+\mathcal{L}_{\text {gauge }}+\mathcal{L}_{D, \theta}+\mathcal{L}_{\Omega}+\mathcal{L}_{\Lambda}+\mathcal{L}_{J}
$$

As a result, by the method of using the operator $\hat{U}$, the $(0,2)$ action of the total Lagrangin density of Eq. (49) agrees exactly with the $(2,2)$ action $S_{(2,2)}$ in [24], because of the correspondences in Eqs. (41), (47), and (48). By using the new operator $\hat{U}$, unlike in the method of Ref. 24] where it is impossible to have different values of $\mathrm{U}(1)$ charges, we could assign different values of $\mathrm{U}(1)$ charges to each local coordinate, and provide a more general $(0,2)$ Lagrangian density. These result will lead to a more general Calabi-Yau supermanifold which has a different number of even local coordinates and odd local coordinates, as will be shown in later sections.

\section{3 (0,2) Supersymmetric Transformations and Invariance of Lagrangian Densities}

In this section we will show the $(0,2)$ supersymmetric transformation properties of the field components, and prove the $(0,2)$ supersymmetric invariances of the Lagrangian densities introduced in section 2 , up to the total derivatives.

The $(2,2)$ supersymmetric transformation property of the total $(2,2)$ Lagrangian density has been indicated in Ref. 24]. However the $(0,2)$ supersymmetric transformation property of the total $(0,2)$ Lagrangian density, in which each local coordinate has the same U(1) charge, has not yet been explicitly indicated. We are able to find the $(0,2)$ supersymmetric transformation property of the $(0,2)$ Lagrangian density by looking at the $(2,2)$ supersymmetric transformation property of the $(2,2)$ Lagrangian density indirectly. This is different from the method of Ref. [24], where the $(0,2)$ supersymmetric transformation property of the superpotential term is calculated indirectly, and the supersymmetric transformation of $U(1)$ vector multiplets and the U(1) gauge transformation is not included. Therefore, by assigning different values of U(1) charges to each local coordinate and assigning the correct $(0,2)$ supersymmetric transformation property to the superpotential term, we define the $(0,2)$ supersymmetric transformation operator by using the new operator $\hat{U}$, and we will verify the $(0,2)$ supersymmetric transformation property of the total $(0,2)$ Lagrangian density.

First, we define an operation of the $(0,2)$ supersymmetric transformations from Eq. (7):

$$
\delta_{(0,2)}=-\epsilon_{-} \mathcal{Q}_{+}+\bar{\epsilon}_{-} \overline{\mathcal{Q}}_{+} .
$$

We can then derive the $(0,2)$ supersymmetric transformation properties of the field components, and can show that they match the $(2,2)$ supersymmetric transformation properties by using the correspondence relations of Eqs. (41), (47), and (48). The $(0,2)$ supersymmetric transformation properties for the field components of the $(2,2)$ Grassmann even chiral superfield are given by

$$
\begin{aligned}
& \delta_{(0,2)} \phi^{I}=-\sqrt{2} \epsilon_{-} \psi_{+}^{I} \\
& \delta_{(0,2)} \psi_{+}^{I}=\sqrt{2} i \bar{\epsilon}_{-} D_{+} \phi^{I}, \\
& \delta_{(0,2)} \psi_{-}^{I}=\sqrt{2}\left(\epsilon_{-} F^{I}+\sqrt{2} \bar{\epsilon}_{-} Q_{I} \sigma \phi^{I}\right), \\
& \delta_{(0,2)} F^{I}=-\sqrt{2} \bar{\epsilon}_{-}\left(i D_{+} \psi_{-}^{I}-\sqrt{2} i Q_{I} \bar{\lambda}_{+} \phi^{I}-\sqrt{2} Q_{I} \psi_{+}^{I} \sigma\right) .
\end{aligned}
$$

In addition, the $(0,2)$ supersymmetric transformation properties for the field components of the $(2,2)$ Grassmann odd chiral superfield are: 


$$
\begin{aligned}
& \delta_{(0,2)} \xi^{A}=-\sqrt{2} \epsilon_{-} b_{+}^{A}, \\
& \delta_{(0,2)} b_{+}^{A}=\sqrt{2} i \bar{\epsilon}_{-} D_{+} \xi^{A} \\
& \delta_{(0,2)} b_{-}^{A}=\sqrt{2}\left(\epsilon_{-} \chi^{A}+\sqrt{2} \bar{\epsilon}_{-} q_{A} \sigma \xi^{A}\right) \\
& \delta_{(0,2)} \chi^{A}=-\sqrt{2} \bar{\epsilon}_{-}\left(i D_{+} b_{-}^{A}-\sqrt{2} i q_{A} \bar{\lambda}_{+} \xi^{A}-\sqrt{2} q_{A} b_{+}^{A} \sigma\right) .
\end{aligned}
$$

The $(0,2)$ supersymmetric transformation properties for the field components of the $(2,2)$ vector superfield are given as follows:

$$
\begin{aligned}
& \delta_{(0,2)} v_{+}=0, \\
& \delta_{(0,2)} v_{-}=2 i\left(\epsilon_{-} \bar{\lambda}_{-}+\bar{\epsilon}_{-} \lambda_{-}\right), \\
& \delta_{(0,2)} \sigma=-\sqrt{2} i \epsilon_{-} \bar{\lambda}_{+}, \\
& \delta_{(0,2)} \lambda_{-}=i \epsilon_{-}\left(D-i v_{03}\right), \\
& \delta_{(0,2)} \lambda_{+}=\sqrt{2} \epsilon_{-} \partial_{+} \bar{\sigma}, \\
& \delta_{(0,2)} D=\epsilon_{-} \partial_{+} \bar{\lambda}_{-}-\bar{\epsilon}_{-} \partial_{+} \lambda_{-},
\end{aligned}
$$

Where $v_{03}=\partial_{0} v_{3}-\partial_{3} v_{0}$.

The $(0,2)$ supersymmetric transformation properties of the Lagrangian densities $\mathcal{L}_{(0,2)}$ are derived and expressed by using the $(2,2)$ field components from Eqs. (41), (47), and (48). The actions for $\mathcal{L}_{(0,2)}$ are supersymmetric invariants up to total derivatives. However, for $\mathcal{L}_{J}$ in Eq. (37), we obtain:

$$
\begin{aligned}
& \delta_{(0,2)} \mathcal{L}_{J} \\
= & \sqrt{2} i \bar{\epsilon}_{-} \partial_{+}\left[\sum_{I} \psi_{-}^{I} \frac{\partial W(\phi, \xi)}{\partial \phi^{I}}+\sum_{A} b_{-}^{A} \frac{\partial W(\phi, \xi)}{\partial \xi^{A}}\right] \\
& -\sqrt{2} \bar{\epsilon}_{-}\left[v_{+} \hat{U}\left(\sum_{I} \psi_{-}^{I} \frac{\partial W(\phi, \xi)}{\partial \phi^{I}}+\sum_{A} b_{-}^{A} \frac{\partial W(\phi, \xi)}{\partial \xi^{A}}\right)\right. \\
& +\left(\sum_{J} \psi_{+}^{J} \frac{\partial}{\partial \phi^{J}}+\sum_{B} b_{+}^{B} \frac{\partial}{\partial \xi^{B}}+i \bar{\lambda}_{+} \frac{\partial}{\partial \sigma}\right)\left(\sum_{I} \sqrt{2} Q_{I} \sigma \phi^{I} \frac{\partial W(\phi, \xi)}{\partial \phi^{I}}\right. \\
& \left.\left.+\sum_{A} \sqrt{2} q_{A} \sigma \xi^{A} \frac{\partial W(\phi, \xi)}{\partial \xi^{A}}\right)\right]+(\text { h.c. }) .
\end{aligned}
$$

The results of Eq. (54) imply that the action for $\mathcal{L}_{J}$ is not a supersymmetric invariant, because the variations consist of non-total derivative terms under the $(0,2)$ supersymmetric transformation.

Therefore, we must impose consistency conditions that will assure that $\mathcal{L}_{J}$ is $(0,2)$ supersymmetric invariant up to total derivatives:

$$
\sum_{I} Q_{I} \phi^{I} \frac{\partial W(\phi, \xi)}{\partial \phi^{I}}+\sum_{A} q_{A} \xi^{A} \frac{\partial W(\phi, \xi)}{\partial \xi^{A}}=0 .
$$

Equation (55) is the same condition as Eq. (35). We undestand that Eq. (35) does not only define $(0,2)$ chiral superfields, but also gives a consistency condition that ensures the supersymmetric variation of $\mathcal{L}_{J}$ to be invariant under $(0,2)$ supersymmetric transformations up to total derivatives.

These restrictions on the superpotential are confirmed by using the corrected $(0,2)$ supersymmetric transformation which includes the U(1) vector multiplets. Using the method of Ref. 24, one could not confirm the necessity of the restrictions clearly. However, we in our present method 
we could indicate explicitly the necessity of the restrictions. It has been reported (Ref. 24]) that Eq. (55) is equivalent to the quasi-homogeneous condition $W\left(\phi^{I}, \xi^{A}\right)=W\left(\lambda^{Q_{I}} \phi^{I}, \lambda^{q_{A}} \xi^{A}\right)$ for the superpotential. Thus, we can use the identification:

$$
\begin{aligned}
\left(\phi^{1}, \phi^{2}, \cdots, \phi^{m} \mid \xi^{1}, \xi^{2}, \cdots, \xi^{n}\right) & \\
& \sim\left(\lambda^{Q_{1}} \phi^{1}, \lambda^{Q_{2}} \phi^{2}, \cdots, \lambda^{Q_{m}} \phi^{m} \mid \lambda^{q_{1}} \xi^{1}, \lambda^{q_{2}} \xi^{2}, \cdots, \lambda^{q_{n}} \xi^{n}\right),
\end{aligned}
$$

where $\lambda \in C^{\times}$. Namely, the supermanifold $\mathcal{M}^{m \mid n}$ becomes the super weighted complex projective space $W C P^{m-1 \mid n}$, which can be reproduced using $\hat{U}$. If we focus on the Calabi-Yau supermanifold corresponding to the super Landau-Ginzburg model, we can construct a CalabiYau supermanifold, which is more general in Ref. 24] and which has different numbers of even coordinates and odd coordinates satisfying Eq. (11).

The formula of the $\hat{U}$ charge operator satisfies the assumptions of Eqs. (4), (5), (15), (23), (27), (33) and (45). The operator $\hat{U}$ is written as follows:

$$
\begin{aligned}
\hat{U}= & \sum_{I} Q_{I}\left[\phi^{I} \frac{\partial}{\partial \phi^{I}}+\sum_{\mu} \partial_{\mu} \phi^{I} \frac{\partial}{\partial\left(\partial_{\mu} \phi^{I}\right)}+\sum_{\mu, \nu} \partial_{\mu} \partial^{\mu} \phi^{I} \frac{\partial}{\partial\left(\partial_{\nu} \partial^{\nu} \phi^{I}\right)}+\sum_{\alpha= \pm}\left\{\psi_{\alpha}^{I} \frac{\partial}{\partial \psi_{\alpha}^{I}}\right.\right. \\
& \left.+\sum_{\mu} \partial_{\mu} \psi_{\alpha}^{I} \frac{\partial}{\partial\left(\partial_{\mu} \psi_{\alpha}^{I}\right)}\right\}+F^{I} \frac{\partial}{\partial F^{I}} \\
& +\sum_{A} q_{A}\left[\xi^{A} \frac{\partial}{\partial \xi^{A}}+\sum_{\mu} \partial_{\mu} \xi^{A} \frac{\partial}{\partial\left(\partial_{\mu} \xi^{A}\right)}+\sum_{\mu, \nu} \partial_{\mu} \partial^{\mu} \xi^{A} \frac{\partial}{\partial\left(\partial_{\nu} \partial^{\nu} \xi^{A}\right)}+\sum_{\alpha= \pm}\left\{b_{\alpha}^{A} \frac{\partial}{\partial b_{\alpha}^{A}}\right.\right. \\
& \left.+\sum_{\mu} \partial_{\mu} b_{\alpha}^{A} \frac{\partial}{\partial\left(\partial_{\mu} b_{\alpha}^{A}\right)}\right\}+\chi^{A} \frac{\partial}{\partial \chi^{A}}+\text { (h.c.). }
\end{aligned}
$$

By using the operator $\hat{U}$, we could assign different values of $\mathrm{U}(1)$ charges to the each local coordinate. Furthermore, different from method of Ref. 24, we could indicate the necessity of the restrictions on the superpotential explicitly, and succeeded in constructing a more general $(0,2)$ Lagrangian density, which has different $\mathrm{U}(1)$ charges for each local coordinate.

\section{4 (0,2) Supermultiplets in the Non-Abelian Gauge Theory}

Now we will construct the $(0,2)$ Lagrangian density for the $\mathrm{U}(\mathrm{N})$ gauge group. In contrast to the $\mathrm{U}(1)$ case, in the $\mathrm{U}(\mathrm{N})$ case we do not need to assign different values of the charge to each local coordinate. By introducing the $(0,2)$ supermultiplets in the $\mathrm{U}(\mathrm{N})$ gauge group, we can construct the $(0,2) \mathrm{U}(\mathrm{N})$ Lagrangian density without using the $\hat{U}$ operator at first.

First, $\Psi=\theta^{+} \bar{\theta}^{+} \sum_{a} v_{+}^{a} T^{a}$ is defined for the vector fields $v_{\mu}$, where $T^{a}$ are the generators of the $\mathrm{U}(\mathrm{N})$ group and $a=1, \cdots, \operatorname{dim} \mathrm{U}(\mathrm{N})$. In $D=2$, the $(2,2)$ Grassmann even chiral superfield $\Phi_{(2,2) i}$ and $(2,2)$ Grassmann odd chiral superfield $\Xi_{(2,2) i}$ are given in a manner similar to the $\mathrm{U}(1)$ case:

$$
\begin{aligned}
& \Phi_{(2,2) i}^{I} \\
= & \phi_{i}^{I}+\sqrt{2}\left(\theta^{+} \psi_{+i}^{I}+\theta^{-} \psi_{-i}^{I}\right)+2 \theta^{+} \theta^{-} F_{i}^{I}-i \theta^{-} \bar{\theta}^{-} \partial_{-} \phi_{i}^{I}-i \theta^{+} \bar{\theta}^{+} \partial_{+} \phi_{i}^{I} \\
& -\sqrt{2} i \theta^{+} \theta^{-} \bar{\theta}^{-} \partial_{-} \psi_{+i}^{I}+\sqrt{2} i \theta^{+} \theta^{-} \bar{\theta}^{+} \partial_{+} \psi_{-i}^{I}-\theta^{+} \theta^{-} \bar{\theta}^{-} \bar{\theta}^{+} \partial_{-} \partial_{+} \phi_{i}^{I}, \\
& \Xi_{(2,2) i}^{A} \\
= & \xi_{i}^{A}+\sqrt{2}\left(\theta^{+} b_{+i}^{A}+\theta^{-} b_{-i}^{A}\right)+2 \theta^{+} \theta^{-} \chi_{i}^{A}-i \theta^{-} \bar{\theta}^{-} \partial_{-} \xi_{i}^{A}-i \theta^{+} \bar{\theta}^{+} \partial_{+} \xi_{i}^{A} \\
& -\sqrt{2} i \theta^{+} \theta^{-} \bar{\theta}^{-} \partial_{-} b_{+i}^{A}+\sqrt{2} i \theta^{+} \theta^{-} \bar{\theta}^{+} \partial_{+} b_{-i}^{A}-\theta^{+} \theta^{-} \bar{\theta}^{-} \bar{\theta}^{+} \partial_{-} \partial_{+} \xi_{i}^{A},
\end{aligned}
$$


where $i=1, \cdots, N$ 24]. For these $(2,2)$ chiral superfields, we will define superfields with restrictions $\theta^{-}=\bar{\theta}^{-}=0$ as follows:

$$
\begin{aligned}
& \left.\Phi_{(0,2) i}^{I} \equiv \sum_{j}\left(e^{\Psi}\right)_{i j} \Phi_{(2,2) j}^{I}\right|_{\theta^{-}=\bar{\theta}^{-}=0}, \\
& \left.\Xi_{(0,2) i}^{A} \equiv \sum_{j}\left(e^{\Psi}\right)_{i j} \Xi_{(2,2) j}^{A}\right|_{\theta^{-}=\bar{\theta}^{-}=0},
\end{aligned}
$$

where the covariant derivatives of the gauge transformation for the components of the $(2,2)$ chiral superfields are defined as:

$$
\left(D_{\mu} \phi^{I}\right)_{i}=\partial_{\mu} \phi_{i}^{I}+i \sum_{j} v_{\mu i j} \phi_{j}^{I} .
$$

We now consider the Lagrangian density $\mathcal{L}_{\text {non.kin. }}$ for the fields in Eqs. (60) and (61). From the definition of the $(2,2)$ vector superfield,

$$
\mathcal{V}=\sum_{a}\left(i v_{-}^{a}+2 \theta^{+} \bar{\lambda}_{-}^{a}+2 \bar{\theta}^{+} \lambda_{-}^{a}+2 i \theta^{+} \bar{\theta}^{+} D^{a}\right) T^{a},
$$

we can define the covariant derivative:

$$
\mathcal{D}_{0}-\mathcal{D}_{3} \equiv \partial_{-}+\mathcal{V}
$$

Then, $\mathcal{L}_{\text {non.kin. }}$ is given by Eqs. (60), (61) and (64)

$$
\begin{aligned}
\mathcal{L}_{\text {non.kin. }}=\frac{i}{2} \int d \theta^{+} d \bar{\theta}^{+} \sum_{i, j} & {\left[\sum_{I} \bar{\Phi}_{(0,2) i}^{I}\left(\mathcal{D}_{0}-\mathcal{D}_{3}\right)_{i j} \Phi_{(0,2) j}^{I}\right.} \\
& \left.+\sum_{A} \bar{\Xi}_{(0,2) i}^{A}\left(\mathcal{D}_{0}-\mathcal{D}_{3}\right)_{i j} \Xi_{(0,2) j}^{A}\right] .
\end{aligned}
$$

The Lagrangian density $\mathcal{L}_{\text {non.gauge }}$ for the vector superfield $\mathcal{V}$ and Fayet-Iliopoulos(FI) term $\mathcal{L}_{\text {non.D, } \theta}$, which arises from $\mathrm{U}(1)$ sector of the $\mathrm{U}(\mathrm{N})$ group, is given as follows. We define an operator acting on a function $f_{i}\left(x_{\mu}, \theta^{+}, \bar{\theta}^{+}\right)$as

$$
\sum_{j} \mathcal{D}_{+i j} f_{j} \equiv \sum_{j, k}\left(e^{-\Psi}\right)_{i k}\left(\frac{\partial}{\partial \theta^{+}}-i \bar{\theta}^{+} \partial_{+}\right)\left(e^{\Psi}\right)_{k j} f_{j} .
$$

From Eqs. (64) and (66), we obtain

$$
\Upsilon_{\text {non. }} \equiv\left[\overline{\mathcal{D}}_{+},\left(\mathcal{D}_{0}-\mathcal{D}_{3}\right)\right] .
$$

The covariant derivatives of the gauge transformations for the components of the $(2,2)$ vector superfield are given by

$$
D_{ \pm} \lambda_{-}=\partial_{ \pm} \lambda_{-}+i\left[v_{ \pm}, \lambda_{-}\right] .
$$

From Eq. [67], $\mathcal{L}_{\text {non.gauge }}$ can be given as

$$
\mathcal{L}_{\text {non.gauge }}=-\frac{1}{8 e^{2}} \int d \theta^{+} d \bar{\theta}^{+} \operatorname{tr}\left[\Upsilon_{\text {non. }} \bar{\Upsilon}_{\text {non. }}\right],
$$


and $\mathcal{L}_{n o n . D, \theta}$ is

$$
\mathcal{L}_{\text {non.D }, \theta}=\left.\frac{t}{4} \int d \theta^{+} \operatorname{tr} \Upsilon_{n o n \cdot}\right|_{\bar{\theta}^{+}=0}+\left.\frac{\bar{t}}{4} \int d \bar{\theta}^{+} \operatorname{tr} \bar{\Upsilon}_{n o n \cdot}\right|_{\theta^{+}=0} .
$$

Since Eq. (67) includes only part of the components of the $(2,2)$ vector superfield, the residual compensating components will be given by the superfield $\Omega$ :

$$
\Omega \equiv \sum_{a}\left(\sigma^{a}+\sqrt{2} i \theta^{+} \bar{\lambda}_{+}^{a}-i \theta^{+} \bar{\theta}^{+} \partial_{+} \sigma^{a}\right) T^{a} .
$$

From Eq. (71), we redefine the following superfield:

$$
\Omega_{n o n .} \equiv \Omega+[\Psi, \Omega]
$$

and from these definitions, we obtain the following:

$$
\begin{aligned}
\mathcal{V}^{\prime} & \equiv \sum_{a}\left(i v_{-}^{a}+2 \sqrt{2} \theta^{+} \bar{\lambda}_{-}^{a}+2 \sqrt{2} \bar{\theta}^{+} \lambda_{-}^{a}+2 i \theta^{+} \bar{\theta}^{+} D^{a}\right) T^{a}, \\
\Omega^{\prime} & \equiv \sum_{a}\left(\sigma^{a}+i \theta^{+} \bar{\lambda}_{+}^{a}-i \theta^{+} \bar{\theta}^{+} \partial_{+} \sigma^{a}\right) T^{a} .
\end{aligned}
$$

Using Eq. (74), we can define

$$
\Omega_{\text {non. }}^{\prime} \equiv \Omega^{\prime}+\left[\Psi, \Omega^{\prime}\right]
$$

From Eqs. (73) and (75),

$$
\left.\Gamma \equiv\left[\mathcal{V}^{\prime}, \Omega_{\text {non. }}^{\prime}\right]\right|_{\bar{\theta}^{+}=0},
$$

is defined. Then, the Lagrangian density $\mathcal{L}_{\text {non.gauge }}^{\prime}$ is obtained from Eqs. (72), (75) and (76):

$$
\begin{gathered}
\mathcal{L}_{\text {non.gauge }}^{\prime}=\frac{i}{2 e^{2}} \int d \theta^{+} d \bar{\theta}^{+} \operatorname{tr}\left[\bar{\Omega}_{n o n .} \partial_{-} \Omega_{\text {non. }}+\bar{\Omega}_{n o n .}^{\prime} \Gamma-\bar{\Gamma} \Omega_{\text {non. }}^{\prime}\right. \\
\left.-i \theta^{+} \bar{\theta}^{+}\left[\Omega_{\text {non. }}^{\prime}, \bar{\Omega}_{n o n .}^{\prime}\right]^{2}\right] .
\end{gathered}
$$

Next, we will introduce the other $(0,2)$ superfields as follows:

$$
\begin{aligned}
& \Lambda_{I i}^{\prime} \equiv \psi_{-i}^{I}-\sqrt{2} \theta^{+} F_{i}^{I}-i \theta^{+} \bar{\theta}^{+} \partial_{+} \psi_{-i}^{I}, \\
& \tilde{\Lambda}_{A i}^{\prime} \equiv b_{-i}^{A}-\sqrt{2} \theta^{+} \chi_{i}^{A}-i \theta^{+} \bar{\theta}^{+} \partial_{+} b_{-i}^{A} .
\end{aligned}
$$

We will give functions $E_{I i}\left(\Phi_{(0,2)}, \Omega\right)$, and $\tilde{E}_{A i}\left(\Xi_{(0,2)}, \Omega\right)$ defined on the variables given by Eqs. (60), (61) and (71). We assume these functions to be separable in variables:

$$
\begin{aligned}
& E_{I i}\left(\Phi_{(0,2)}, \Omega\right)=\sum_{j} H_{i j}(\Omega) G_{I j}\left(\Phi_{(0,2)}\right), \\
& \tilde{E}_{A i}\left(\Xi_{(0,2)}, \Omega\right)=\sum_{j} H_{i j}(\Omega) \tilde{G}_{A j}\left(\Xi_{(0,2)}\right) .
\end{aligned}
$$

Using Eqs. (78)-(81), we redefine the fields:

$$
\begin{aligned}
& \Lambda_{I i} \equiv \Lambda_{I i}^{\prime}-\sqrt{2} \bar{\theta}^{+} E_{I i}\left(\Phi_{(0,2)}, \Omega\right), \\
& \tilde{\Lambda}_{A i} \equiv \tilde{\Lambda}_{A i}^{\prime}-\sqrt{2} \bar{\theta}^{+} \tilde{E}_{A i}\left(\Xi_{(0,2)}, \Omega\right) .
\end{aligned}
$$


We can then obtain the following identities from Eqs. (80)-83):

$$
\begin{aligned}
& \Lambda_{I i(0,2)} \equiv \sum_{j}\left(e^{\Psi}\right)_{i j} \Lambda_{I j}, \\
& \tilde{\Lambda}_{A i(0,2)} \equiv \sum_{j}\left(e^{\Psi}\right)_{i j} \tilde{\Lambda}_{A j},
\end{aligned}
$$

and

$$
\begin{aligned}
& E_{I i}^{\prime}\left(\Phi_{(0,2)} \bar{\Omega}\right)=\sum_{j} \bar{H}_{i j}(\bar{\Omega}) G_{I j}\left(\Phi_{(0,2)}\right), \\
& \tilde{E}_{A i}^{\prime}\left(\Xi_{(0,2)}, \bar{\Omega}\right)=\sum_{j} \bar{H}_{i j}(\bar{\Omega}) \tilde{G}_{A j}\left(\Xi_{(0,2)}\right) .
\end{aligned}
$$

We can now obtain the Lagrangian density $\mathcal{L}_{\text {non. } \Lambda}$ from Eqs. (80), (81), and (84)-(87):

$$
\begin{aligned}
\mathcal{L}_{\text {non. } . \Lambda} & \frac{1}{2} \int d \theta^{+} d \bar{\theta}^{+} \sum_{i}\left[\sum_{I} \bar{\Lambda}_{I i(0,2)} \Lambda_{I i(0,2)}+\sum_{A} \overline{\tilde{\Lambda}}_{A i(0,2)} \tilde{\Lambda}_{A i(0,2)}\right. \\
& +\sum_{I}\left(\bar{\theta}^{+} \bar{E}_{I i}\left(\bar{\Phi}_{(0,2)}, \bar{\Omega}\right)+\theta^{+} \bar{E}_{I i}^{\prime}\left(\bar{\Phi}_{(0,2)}, \Omega\right)\right) \\
& \times\left(\theta^{+} E_{I i}\left(\Phi_{(0,2)}, \Omega\right)+\bar{\theta}^{+} E_{I i}^{\prime}\left(\Phi_{(0,2)}, \bar{\Omega}\right)\right) \\
& -\sum_{A}\left(\bar{\theta}^{+} \overline{\tilde{E}}_{A i}\left(\bar{\Xi}_{(0,2)}, \bar{\Omega}\right)+\theta^{+} \overline{\tilde{E}}_{A i}^{\prime}\left(\bar{\Xi}_{(0,2)}, \Omega\right)\right) \\
& \left.\times\left(\theta^{+} \tilde{E}_{A i}\left(\Xi_{(0,2)}, \Omega\right)+\bar{\theta}^{+} \tilde{E}_{A i}^{\prime}\left(\Xi_{(0,2)}, \bar{\Omega}\right)\right)\right] .
\end{aligned}
$$

We will choose the functions given in Eqs. (800) and (81) as

$$
\begin{aligned}
& G_{I i}(\phi)=\sqrt{2} \phi_{i}^{I}, \\
& \tilde{G}_{A i}(\xi)=\sqrt{2} \xi_{i}^{A}, \\
& H(\sigma)=\sigma .
\end{aligned}
$$

We further define functions $J_{i}^{I}\left(\Phi_{(0,2)}, \Xi_{(0,2)}\right)$ and $\tilde{J}_{i}^{A}\left(\Phi_{(0,2)}, \Xi_{(0,2)}\right)$ by Eqs. (60) and (61), and assume the following relations:

$$
\begin{gathered}
J_{i}^{I}(\phi, \xi)=\frac{\partial W(\phi, \xi)}{\partial \phi_{i}^{I}}, \\
\tilde{J}_{i}^{A}(\phi, \xi)=\frac{\partial W(\phi, \xi)}{\partial \xi_{i}^{A}},
\end{gathered}
$$

where $W$ is superpotential of the theory. The Lagrangian density $\mathcal{L}_{\text {non.J }}$ is then obtained by Eqs. (84), (85) and (90) as follows:

$$
\begin{aligned}
& \mathcal{L}_{\text {non.J }} \\
& =\frac{1}{\sqrt{2}} \int d \theta^{+} \sum_{i}\left[\sum_{I} \Lambda_{I i(0,2)} J_{i}^{I}\left(\Phi_{(0,2)}, \Xi_{(0,2)}\right)\right. \\
& \left.+\sum_{A} \tilde{\Lambda}_{A i(0,2)} \tilde{J}_{i}^{A}\left(\Phi_{(0,2)}, \Xi_{(0,2)}\right)\right]\left.\right|_{\bar{\theta}^{+}=0} \\
& +\frac{1}{\sqrt{2}} \int d \bar{\theta}^{+} \sum_{i}\left[\sum_{I} \bar{J}_{i}^{I}\left(\bar{\Phi}_{(0,2)}, \bar{\Xi}_{(0,2)}\right) \bar{\Lambda}_{I i(0,2)}\right.
\end{aligned}
$$




$$
\left.+\sum_{A} \overline{\tilde{J}}_{i}^{A}\left(\bar{\Phi}_{(0,2)}, \bar{\Xi}_{(0,2)}\right) \overline{\tilde{\Lambda}}_{A i(0,2)}\right]\left.\right|_{\theta^{+}=0} .
$$

Finally, by combining Eqs. (65), (69), (70), (77), (88), and (91), we can obtain the $(0,2)$ total Lagrangian density $\mathcal{L}_{(0,2) \text { non. }}$ :

$$
\begin{aligned}
\mathcal{L}_{(0,2) \text { non. }}= & \mathcal{L}_{\text {non.kin. }}+\mathcal{L}_{\text {non.gauge }}+\mathcal{L}_{\text {non.D }, \theta}+\mathcal{L}_{\text {non.gauge }}^{\prime} \\
& +\mathcal{L}_{\text {non. } \Lambda}+\mathcal{L}_{\text {non.J. }}
\end{aligned}
$$

In Eq. (92), the $(0,2) \mathrm{U}(\mathrm{N})$ Lagrangian density was constructed without using $\hat{U}$ at this moment, because we do not need to assign differenent values of charge to each local coordinate.

\section{5 (0,2) Supersymmetric Transformation and Invariance of Lagrangian Densities in Non-Abelian Gauge Theory}

In this section, we will verify the $(0,2)$ supersymmetric transformation properties of Eqs. (65), (69), (70), (77), (88), and (91).

In constructing the $(0,2) \mathrm{U}(\mathrm{N})$ Lagrangian density, it appears that similar restrictions on the superpotential are required as in the $\mathrm{U}(1)$ case. So here we concentrate on the restrictions on the superpotential, and compare them for the $\mathrm{U}(\mathrm{N})$ and the $\mathrm{U}(1)$ cases. While for the $\mathrm{U}(1)$ part, a Calabi-Yau supermanifold with the same number of even coordinates and odd coordinates may be obtained, for the $\mathrm{SU}(\mathrm{N})$ part constraints, a supermanifold may be defined which is different from the $\mathrm{U}(1)$ case.

The $(0,2)$ supersymmetric transformation properties of the components of the $(2,2)$ Grassmann even and odd superfields are given, respectively, as follows:

$$
\begin{aligned}
& \delta_{(0,2)} \phi_{i}^{I}=-\sqrt{2} \epsilon_{-} \psi_{+i}^{I}, \\
& \delta_{(0,2)} \psi_{+i}^{I}=\sqrt{2} i \bar{\epsilon}_{-}\left(D_{+} \phi^{I}\right)_{i}, \\
& \delta_{(0,2)} \psi_{-i}^{I}=\sqrt{2} \epsilon_{-} F_{i}^{I}+2 \bar{\epsilon}_{-} \sum_{j} \sigma_{i j} \phi_{j}^{I}, \\
& \delta_{(0,2)} F_{i}^{I}=\bar{\epsilon}_{-}\left\{-\sqrt{2} i\left(D_{+} \psi_{-}^{I}\right)_{i}+2 \sum_{j} \sigma_{i j} \psi_{+j}^{I}+2 i \sum_{j} \bar{\lambda}_{+i j} \phi_{j}^{I}\right\} \\
& \delta_{(0,2)} \xi_{i}^{A}=-\sqrt{2} \epsilon_{-} b_{+i}^{A}, \\
& \delta_{(0,2)} b_{+i}^{A}=\sqrt{2} i \bar{\epsilon}_{-}\left(D_{+} \xi^{A}\right)_{i}, \\
& \delta_{(0,2)} b_{-i}^{A}=\sqrt{2} \epsilon_{-} \chi_{i}^{A}+2 \bar{\epsilon}_{-} \sum_{j} \sigma_{i j} \xi_{j}^{A}, \\
& \delta_{(0,2)} \chi_{i}^{A}=\bar{\epsilon}_{-}\left\{-\sqrt{2} i\left(D_{+} b_{-}^{A}\right)_{i}+2 \sum_{j} \sigma_{i j} b_{+j}^{A}+2 i \sum_{j} \bar{\lambda}_{+i j} \xi_{j}^{A}\right\} .
\end{aligned}
$$

The $(0,2)$ supersymmetric transformation properties of components of the $(2,2)$ vector superfield are given as:

$$
\begin{aligned}
& \delta_{(0,2)} v_{+}=0 \\
& \delta_{(0,2)} v_{-}=2 i\left(\epsilon_{-} \bar{\lambda}_{-}+\bar{\epsilon}_{-} \lambda_{-}\right), \\
& \delta_{(0,2)} \sigma=-\sqrt{2} i \epsilon_{-} \bar{\lambda}_{+}, \\
& \delta_{(0,2)} \lambda_{-}=i \epsilon_{-}\left(D-i v_{03 n o n .}-[\sigma, \bar{\sigma}]\right), \\
& \delta_{(0,2)} \lambda_{+}=\sqrt{2} \epsilon_{-} D_{+} \bar{\sigma}, \\
& \delta_{(0,2)} D=\epsilon_{-}\left(D_{+} \bar{\lambda}_{-}+\sqrt{2} i\left[\bar{\sigma}, \bar{\lambda}_{+}\right]\right)-\bar{\epsilon}_{-}\left(D_{+} \lambda_{-}+\sqrt{2} i\left[\sigma, \lambda_{+}\right]\right),
\end{aligned}
$$

where $v_{03 n o n .}=\partial_{0} v_{3}-\partial_{3} v_{0}+i\left[v_{0}, v_{3}\right]$. 


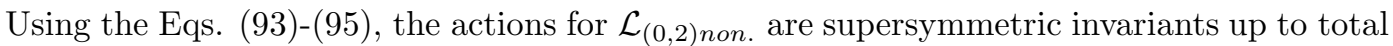
derivatives. However, for $\mathcal{L}_{\text {non.J }}$ in Eq. (91), we obtain:

$$
\begin{aligned}
& \delta_{(0,2)} \mathcal{L}_{\text {non.J }} \\
= & \sqrt{2} i \bar{\epsilon}_{-} \partial_{+} \sum_{i}\left[\sum_{I} \psi_{-i}^{I} \frac{\partial W(\phi, \xi)}{\partial \phi_{i}^{I}}+\sum_{A} b_{-i}^{A} \frac{\partial W(\phi, \xi)}{\partial \xi_{i}^{A}}\right] \\
& -\sqrt{2} \bar{\epsilon}_{-} \sum_{i, j}\left[\sum_{k, J} v_{+i j} \psi_{-k}^{J} \frac{\partial}{\partial \phi_{k}^{J}}+\sqrt{2} \sum_{k, J} \sigma_{i j} \psi_{+k}^{J} \frac{\partial}{\partial \phi_{k}^{J}}+\sum_{k, B} v_{+i j} b_{-k}^{B} \frac{\partial}{\partial \xi_{k}^{B}}\right. \\
& \left.+\sqrt{2} \sum_{k, B} \sigma_{i j} b_{+k}^{B} \frac{\partial}{\partial \xi_{k}^{B}}+\sqrt{2} i \bar{\lambda}_{+i j}\right]\left[\sum_{I} \phi_{j}^{I} \frac{\partial W(\phi, \xi)}{\partial \phi_{i}^{I}}+\sum_{A} \xi_{j}^{A} \frac{\partial W(\phi, \xi)}{\partial \xi_{i}^{A}}\right]+\text { (h.c.). }
\end{aligned}
$$

Next, we derive the consistency condition for the $(0,2)$ supersymmetric invariances of the action under the $(0,2)$ supersymmetric transformation by the following relation using Eq. (96):

$$
\sum_{i, j}\left(\sum_{I} T_{i j}^{a} \phi_{j}^{I} \frac{\partial W(\phi, \xi)}{\partial \phi_{i}^{I}}+\sum_{A} T_{i j}^{a} \xi_{j}^{A} \frac{\partial W(\phi, \xi)}{\partial \xi_{i}^{A}}\right)=0 .
$$

We define Eq.97) as a function $G(\phi, \xi)$ :

$$
G(\phi, \xi) \equiv \sum_{i, j}\left(\sum_{I} T_{i j}^{a} \phi_{j}^{I} \frac{\partial W(\phi, \xi)}{\partial \phi_{i}^{I}}+\sum_{A} T_{i j}^{a} \xi_{j}^{A} \frac{\partial W(\phi, \xi)}{\partial \xi_{i}^{A}}\right)=0,
$$

and transform the function $G(\phi, \xi)$ under the transformation laws:

$$
\phi_{i}^{I} \rightarrow \sum_{j}\left(\lambda^{T^{a}}\right)_{i j} \phi_{j}^{I}, \quad \xi_{i}^{A} \rightarrow \sum_{j}\left(\lambda^{T^{a}}\right)_{i j} \xi_{j}^{A} .
$$

Because $G(\phi, \xi)$ is equal to zero, the function transformed by using Eq.(99) also vanishes:

$$
G(\phi, \xi)=G\left(\lambda^{T} \phi, \lambda^{T} \xi\right)=0 .
$$

Eq.(100) gives the equivalence relation for local coordinates in a supermanifold $\mathcal{M}^{m \mid n}$ :

$$
\begin{aligned}
\left(\phi_{i}^{1}, \cdots, \phi_{i}^{m} \mid \xi_{i}^{1}, \cdots, \xi_{i}^{n}\right) & \\
& \sim\left(\sum_{j}\left(\lambda^{T^{a}}\right)_{i j} \phi_{j}^{1}, \cdots, \sum_{j}\left(\lambda^{T^{a}}\right)_{i j} \phi_{j}^{m} \mid \sum_{j}\left(\lambda^{T^{a}}\right)_{i j} \xi_{j}^{1}, \cdots, \sum_{j}\left(\lambda^{T^{a}}\right)_{i j} \xi_{j}^{n}\right) .
\end{aligned}
$$

Eq.(101) may indicate that the manifold $\mathcal{M}^{m \mid n}$ can be considered to be equivalent to the super weighted projective space $W C P^{m-1 \mid n}$. Moreover, we calculate for the superpotential $W\left(\lambda^{T} \phi, \lambda^{T} \xi\right)$ as follows:

$$
\begin{aligned}
& \sum_{i, j}\left(\sum_{I} T_{i j}^{a} \phi_{j}^{I} \frac{\partial W\left(\lambda^{T} \phi, \lambda^{T} \xi\right)}{\partial \phi_{i}^{I}}+\sum_{A} T_{i j}^{a} \xi_{j}^{A} \frac{\partial W\left(\lambda^{T} \phi, \lambda^{T} \xi\right)}{\partial \xi_{i}^{A}}\right) \\
= & \sum_{b, i, j, k, m}\left(\sum_{I} T_{i j}^{a} \phi_{j}^{I}\left(\lambda^{T^{b}}\right)_{k i} \frac{\partial W\left(\lambda^{T} \phi, \lambda^{T} \xi\right)}{\partial\left(\left(\lambda^{T^{b}}\right)_{k m} \phi_{m}^{I}\right)}+\sum_{A} T_{i j}^{a} \xi_{j}^{A}\left(\lambda^{T^{b}}\right)_{k i} \frac{\partial W\left(\lambda^{T} \phi, \lambda^{T} \xi\right)}{\partial\left(\left(\lambda^{T^{b}}\right)_{k m} \xi_{m}^{A}\right)}\right) .
\end{aligned}
$$

Eq. (102) can be divided into the $\mathrm{U}(1)$ part and the $\mathrm{SU}(\mathrm{N})$ part of the $\mathrm{U}(\mathrm{N})$ gauge group. Then, the generators of the $\mathrm{U}(1)$ part and the $\mathrm{SU}(\mathrm{N})$ part are defined as $T_{i j}^{0}=M \delta_{i j}$ and $T_{i j}^{\Theta}$ 
$\left(\Theta=1, \cdots, \mathrm{N}^{2}-1\right)$ respectively, where $M$ is a normalization factor. The $\mathrm{U}(1)$ part of Eq. (102) is calculated as follows:

$$
M \sum_{b, i, j, k}\left(\sum_{I}\left(\lambda^{T^{b}}\right)_{i j} \phi_{j}^{I} \frac{\partial W\left(\lambda^{T} \phi, \lambda^{T} \xi\right)}{\partial\left(\left(\lambda^{T^{b}}\right)_{i k} \phi_{k}^{I}\right)}+\sum_{A}\left(\lambda^{T^{b}}\right)_{i j} \xi_{j}^{A} \frac{\partial W\left(\lambda^{T} \phi, \lambda^{T} \xi\right)}{\partial\left(\left(\lambda^{T^{b}}\right)_{i k} \xi_{k}^{A}\right)}\right)
$$

which coincides with the U(1) part of $G\left(\lambda^{T} \phi, \lambda^{T} \xi\right)$. Furthermore, by using Eq. (100), we found that these equations also coincide with that of $G(\phi, \xi)$ :

$$
\begin{aligned}
\left.G(\phi, \xi)\right|_{a=0} & =\left.G\left(\lambda^{T} \phi, \lambda^{T} \xi\right)\right|_{a=0} \\
& =M \sum_{i}\left(\sum_{I} \phi_{i}^{I} \frac{\partial W(\phi, \xi)}{\partial \phi_{i}^{I}}+\sum_{A} \xi_{i}^{A} \frac{\partial W(\phi, \xi)}{\partial \xi_{i}^{A}}\right) \\
& =M \sum_{i}\left(\sum_{I} \phi_{i}^{I} \frac{\partial W\left(\lambda^{T} \phi, \lambda^{T} \xi\right)}{\partial \phi_{i}^{I}}+\sum_{A} \xi_{i}^{A} \frac{\partial W\left(\lambda^{T} \phi, \lambda^{T} \xi\right)}{\partial \xi_{i}^{A}}\right) \\
& =0 .
\end{aligned}
$$

Therefore, Eq.(104) gives the quasi-homogeneous condition $W(\phi, \xi)=W\left(\lambda^{T} \phi, \lambda^{T} \xi\right)$ for the superpotential.

On the other hand, the $\mathrm{SU}(\mathrm{N})$ part of Eq.(102) is:

$$
\sum_{b, i, j, k, l}\left(\sum_{I}\left(\lambda^{T^{b}}\right)_{i j} T_{j k}^{\Theta} \phi_{k}^{I} \frac{\partial W\left(\lambda^{T} \phi, \lambda^{T} \xi\right)}{\partial\left(\left(\lambda^{T^{b}}\right)_{i l} \phi_{l}^{I}\right)}+\sum_{A}\left(\lambda^{T^{b}}\right)_{i j} T_{j k}^{\Theta} \xi_{k}^{A} \frac{\partial W\left(\lambda^{T} \phi, \lambda^{T} \xi\right)}{\partial\left(\left(\lambda^{T^{b}}\right)_{i l} \xi_{l}^{A}\right)}\right)
$$

which, however, does not coincide with the $\mathrm{SU}(\mathrm{N})$ part of $G(\phi, \xi)$ and of $G\left(\lambda^{T} \phi, \lambda^{T} \xi\right)$. Thus the superpotential $W(\phi, \xi)$ does not satisfy a quasi-homogeneous condition in $\mathrm{SU}(\mathrm{N})$.

From these results, the supermanifold $\mathcal{M}^{m \mid n}$ seems to become the super weighted complex projective space $W C P^{m-1 \mid n}$, although the superpotential $W(\phi, \xi)$ for the non-Abelian gauge group does not satisfy a quasi-homogeneous condition in $\mathrm{SU}(\mathrm{N})$, except for the $\mathrm{U}(1)$ part of $\mathrm{U}(\mathrm{N})$. Therefore, because of the extention to the $\mathrm{U}(\mathrm{N})$ gauge group, there are more stringent restrictions to be imposed on the form of the superpotential than in the $\mathrm{U}(1)$ case.

From the U(1) part, the Calabi-Yau supermanifold must have the same number of even coordinates and odd coordinates from Eq. (1). In the $\mathrm{SU}(\mathrm{N})$ part, we must take care in constructing the Calabi-Yau supermanifold, because there are more stringent restrictions to be imposed on the form of the superpotential than in the $\mathrm{U}(1)$ case.

\section{$6 \quad \mathrm{U}(\mathrm{N})$ Charge Operator}

In constructing the $(0,2) \mathrm{U}(\mathrm{N})$ Lagrangian density, we could not confirm the reason of necessity to introduce the $\hat{U}$-type operator. However, in order to introduce the $(0,2)$ chiral superfields, we need this operator, because otherwise we cannot define the $(0,2)$ chirality conditions of the $(0,2)$ chiral superfieldsm as will be shown later. Therefore, in this section, we will introduce the $\hat{U}^{a}$ operator in $\mathrm{U}(\mathrm{N})$ version in order to define the $(0,2)$ chirality conditions of the $(0,2)$ chiral superfields.

We can define the $\mathrm{U}(\mathrm{N})$ charge operator in a manner similar to that for the $\mathrm{U}(1)$ Abelian case:

$$
\hat{U}_{i j}^{a} \equiv \frac{1}{N} \sum_{k, I}\left[T_{i k}^{a} \phi_{k}^{I} \frac{\partial}{\partial \phi_{j}^{I}}+\sum_{\mu} T_{i k}^{a} \partial_{\mu} \phi_{k}^{I} \frac{\partial}{\partial\left(\partial_{\mu} \phi_{j}^{I}\right)}+\sum_{\mu, \nu} T_{i k}^{a} \partial_{\mu} \partial^{\mu} \phi_{k}^{I} \frac{\partial}{\partial\left(\partial_{\nu} \partial^{\nu} \phi_{j}^{I}\right)}\right.
$$




$$
\begin{aligned}
& \left.+\sum_{\alpha} T_{i k}^{a} \psi_{\alpha k}^{I} \frac{\partial}{\partial \psi_{\alpha j}^{I}}+\sum_{\mu, \alpha} T_{i k}^{a} \partial_{\mu} \psi_{\alpha k}^{I} \frac{\partial}{\partial\left(\partial_{\mu} \psi_{\alpha j}^{I}\right)}+T_{i k}^{a} F_{k}^{I} \frac{\partial}{\partial F_{j}^{I}}\right] \\
& +\frac{1}{N} \sum_{k, A}\left[T_{i k}^{a} \xi_{k}^{A} \frac{\partial}{\partial \xi_{j}^{A}}+\sum_{\mu} T_{i k}^{a} \partial_{\mu} \xi_{k}^{A} \frac{\partial}{\partial\left(\partial_{\mu} \xi_{j}^{A}\right)}+\sum_{\mu, \nu} T_{i k}^{a} \partial_{\mu} \partial^{\mu} \xi_{k}^{A} \frac{\partial}{\partial\left(\partial_{\nu} \partial^{\nu} \xi_{j}^{A}\right)}\right. \\
& \left.+\sum_{\alpha} T_{i k}^{a} b_{\alpha k}^{A} \frac{\partial}{\partial b_{\alpha j}^{A}}+\sum_{\mu, \alpha} T_{i k}^{a} \partial_{\mu} b_{\alpha k}^{A} \frac{\partial}{\partial\left(\partial_{\mu} b_{\alpha j}^{A}\right)}+T_{i k}^{a} \chi_{k}^{A} \frac{\partial}{\partial \chi_{j}^{A}}\right]+(\text { h.c. }) .
\end{aligned}
$$

From Eq. (106), the consistency condition in Eq. (97) is rewritten as:

$$
N \sum_{i, j} \delta_{i j} \hat{U}_{i j}^{a} W(\phi, \xi)=0
$$

Using the operator in Eq. (106), we are able to define an operation on the function $f_{i}\left(x_{\mu}, \theta^{+}, \bar{\theta}^{+}\right)$as follows:

$$
\sum_{j} \mathcal{D}_{+i j}^{\prime} f_{j} \equiv \sum_{j, k}\left(e^{-\Psi^{\prime}}\right)_{i k}\left(\frac{\partial}{\partial \theta^{+}}-i \bar{\theta}^{+} \partial_{+}\right)\left(e^{\Psi^{\prime}}\right)_{k j} f_{j},
$$

where $\Psi^{\prime}=\theta^{+} \bar{\theta}^{+} \sum_{a} v_{+}^{a} \hat{U}^{a}$ is assumed. We finally obtain the $(0,2)$ chirality conditions by using Eqs. (60), (61) and (108):

$$
\begin{aligned}
& \sum_{j} \overline{\mathcal{D}}_{+i j}^{\prime} \Phi_{(0,2) j}^{I}=\sum_{i} \mathcal{D}_{+i j}^{\prime} \bar{\Phi}_{(0,2) i}^{I}=0, \\
& \sum_{j} \overline{\mathcal{D}}_{+i j}^{\prime} \Xi_{(0,2) j}^{A}=\sum_{i} \mathcal{D}_{+i j}^{\prime} \Xi_{(0,2) i}^{A}=0 .
\end{aligned}
$$

From these results, we could confirm the necessity of the $\hat{U}$ operator for defining the $(0,2)$ chirality conditions of $(0,2)$ chiral superfields, though this operator was not required for the construction of the $(0,2) \mathrm{U}(\mathrm{N})$ Lagrangian density.

\section{Summary and Discussion}

We have constructed the $D=2,(0,2) \mathrm{U}(1)$ gauged linear sigma model on a supermanifold $\mathcal{M}^{m \mid n}$ by a method which differs from that of Ref. 24], because to our opinion that method seems to be incomplete. Furthermore, we have constructed the $\mathrm{U}(\mathrm{N})$ gauged linear sigma model explicitly.

In the first part of the present paper, we consistently constructed the $D=2,(0,2) \mathrm{U}(1)$ gauged linear sigma model on the supermanifold $\mathcal{M}^{m \mid n}$, by introducing a new operator, $\hat{U}$. In the method of Ref. 24, it was impossible to assign different value of $\mathrm{U}(1)$ charge to each local coordinate. The explicit form of the $\hat{U}$ operator was determined by assuming that it is the operator that assigns different value of $\mathrm{U}(1)$ charges to each local coordinate of $\mathcal{M}^{m \mid n}$. The covariant derivatives and super charges of the $(0,2)$ supersymmetric transformation are also defined using the $\hat{U}$ operator. The $(0,2)$ chirality conditions on the of the superpotential term in the Lagrangian density appear to be most appropriately implied by these covariant derivatives.

The $(0,2)$ supersymmetric invariance of the Lagrangian density of the $(0,2) \mathrm{U}(1)$ gauged linear sigma model was also proved by using consistency conditions derived by using the $\hat{U}$ operator. We found that the conditions that assure the $(0,2)$ supersymmetric invariance of the Lagrangian density agree with the $(0,2)$ chirality conditions for the superpotential. Though the method of Ref. 24] could not confirm the necessity of ristriction conditions clearly, we could 
indicate the necessity of conditions explicitly. The supermanifold $\mathcal{M}^{m \mid n}$ then becomes the super weighted complex projective space $W C P^{m-1 \mid n}$ from these conditions. If we focus on the Calabi-Yau supermanifold corresponding to the super Landau-Ginzburg model, by using $\hat{U}$ we can construct a Calabi-Yau supermanifold which is more general than in Ref.[24], and which has a different number of even coordinates and odd coordinates.

In the second part of the present paper, we constructed a $D=2,(0,2) \mathrm{U}(\mathrm{N})$ gauged linear sigma model on the supermanifold $\mathcal{M}^{m \mid n}$ as a new construction. The construction is approximately parallel to the $\mathrm{U}(1)$ case, but the $\hat{U}^{a}$ operator, which is an extension of the $\hat{U}$ operator of the $\mathrm{U}(1)$ gauge group to the $\mathrm{U}(\mathrm{N})$ gauge group, coincides with a set of generators of $\mathrm{U}(\mathrm{N})$. Although $\hat{U}^{a}$ is unnecessary in constructing the Lagrangian density of the $(0,2) \mathrm{U}(\mathrm{N})$ gauged linear sigma model, we could confirm the necessity of $\hat{U}^{a}$ for giving the $(0,2)$ chirality conditions of the $(0,2)$ chiral superfields. We obtained the conditions that give $(0,2)$ supersymmetric invariance of the Lagrangian density of the $(0,2) \mathrm{U}(\mathrm{N})$ gauged linear sigma model.

As in the case of $U(1)$, these conditions decide the form of the superpotential. However, in the $\mathrm{U}(\mathrm{N})$ case more stringent restrictions on the form of the superpotential have to be imposed than in the $\mathrm{U}(1)$ case. From these results, the superpotential $W(\phi, \xi)$ does not satisfy a quasihomogeneous condition for $\mathrm{SU}(\mathrm{N})$. However, one can argue that the supermanifold $\mathcal{M}^{m \mid n}$ may be a kind of super weighted projective space both for the $\mathrm{U}(1)$ gauged linear sigma model and the $\mathrm{U}(\mathrm{N})$ gauged one.

In the U(1) part, the Calabi-Yau supermanifold must have the same number of even coordinates and odd coordinates from Eq. (11). In the $\mathrm{SU}(\mathrm{N})$ part, we must take care to constructing the Calabi-Yau supermanifold. Because of the more stringent conditions to be imposed on the form of the superpotential than in the $\mathrm{U}(1)$ case, it seems define a certain kind of new supermanifold other than $W C P^{m-1 \mid n}$, which we cannot identify exactly among mathematically defined objects. In our forthcoming paper, we intend to investigate the relationships between the non-linear sigma model and $(0,2)$ linear sigma model in order to investigate further the correspondence with the super Landau-Ginzburg theory. Then, we expect to establish the correspondence between the $D=2,(0,2)$ gauged linear sigma model in the $\mathrm{U}(1)$ and $\mathrm{U}(\mathrm{N})$ gauge groups on the supermanifold to the super Landau-Ginzburg model at $r \ll 0$, which has been reported in the $D=2,(2,2) \mathrm{U}(1)$ gauged linear sigma model 21, 27. As a second step, we hope to investigate the Calabi-Yau supermanifold on the constructed $\mathrm{U}(1)$ and $\mathrm{U}(\mathrm{N})$ gauged linear sigma model mathematically [28, 29, by using the super Landau-Ginzburg mirror symmetry.

\section{References}

[1] E. Witten, Commun. Math. Phys. 252, 189 (2004).

[2] F. Cachazo, P.Svrček and E. Witten, JHEP. 0409, 006 (2004).

[3] C.-J. Zhu, JHEP 0404, 032 (2004).

[4] G. Georgiou, E.W.N. Glover and V.V. Khoze, JHEP 0407, 048 (2004).

[5] A. Brandhuber, B. Spence and G. Travaglini, Nucl. Phys. B 706, 150 (2005).

[6] J. Bedford, A. Brandhuber, B. Spence and G. Travaglini, Nucl. Phys. B 706, 100 (2005).

[7] J. Bedford, A. Brandhuber, B. Spence and G. Travaglini, Nucl. Phys. B 712, 59 (2005).

[8] R. Britto, F. Cachazo, B. Feng and E.Witten, Phys. Rev. Lett. 94, 181602 (2005).

[9] K. Risager, JHEP 0512, 003 (2005).

[10] J.-B. Wu and C.-J. Zhu, JHEP 0407, 032 (2004). 
[11] R. Roiban, M. Spradlin and A. Volovich, Phys. Rev. D 70, 026009 (2004).

[12] E. Witten, Adv. Theor. Math. Phys. 8, 779 (2004).

[13] G. Georgiou and V.V. Khoze, JHEP 0405, 070 (2004).

[14] C. Quigley and M. Rozali, JHEP 0501, 053 (2005).

[15] J.-B. Wu and C.-J. Zhu, JHEP 0409, 063 (2004).

[16] L.J. Dixon, E.W.N. Glover and V.V. Khoze, JHEP 0412, 015 (2004).

[17] Z. Bern, D. Forde, D.A. Kosower and P. Mastrolia, Phys. Rev. D 72, 025006 (2005).

[18] A. Brandhuber, B. Spence and G. Travaglini, JHEP 0702, 088 (2007).

[19] M. Rocek and N. Wadhwa, Adv. Theor. Math. Phys. 9, 315 (2005).

[20] M. Rocek and N. Wadhwa, On Calabi-Yau Supermanifold II, hep-th/0410081.

[21] M. Aganagic and C. Vafa, Mirror Symmetry and Supermanifolds, hep-th/0403192.

[22] C. Ahn, Mirror Symmetry of Calabi-Yau Supermanifolds, hep-th/0407009.

[23] S. P. Kumar and G. Policastro, Phys. Lett. B 619, 163 (2005).

[24] S.Seki and K.Sugiyama, Gauged Linear Sigma Model on Supermanifold, hep-th/0503074.

[25] A. Belhaj, L. B. Drissi, J. Rasmussen, E. H. Saidi and A. Sebbar, Toric Calabi-Yau supermanifolds and mirror symmetry, hep-th/0410291.

[26] J.Wess and J.Bagger, Supersymmetry and Supergravity, (Princeton University Press, 1976).

[27] E. Witten, Nucl. Phys. B 403, 159 (1993).

[28] P. A. Grassi and M. Marescotti, Integration of Superforms and Super-Thom Class, arXiv:0712.2600

[29] R. Catenacci, M. Debernardi, P. A. Grassi and D. Matessi, Balanced Superprojective Varieties, arXiv:0707.4246, 